\title{
Fístula carótido-cavernosa: importancia de su diagnóstico y tratamiento oportunos para prevenir la ceguera
}

\author{
Raimundo Charlin E, Patricio Pacheco B, \\ Francisco Villarroel W, Fernando Urbina A. \\ Carotid cavernous fistula. \\ Report of two cases
}

A carotid cavernous fistula is an abnormal communication between a branch of the carotid artery and the cavernous sinus generating an arteriovenous fistula that can cause severe ocular damage. We hereby report two cases of carotid cavernous fistulas. A 69-year-old male with a 2-month history of right eyelid swelling and red eye aggravated by diplopia, exophthalmus, and limitation of ocular movements one week before consulting. On admisson his visual acuity in the right eye was good (0.5) and biomicroscopy showed arterialization of conjunctival vessels. An orbital CAT scan showed a dilatation of the superior orbital vein characteristic of carotid cavernous fistula. A balloon tamponade of the fistula was performed too late (after 3 weeks) and the eye went blind. A 39-year-old female consulted for an acute proptosis of the right eye and severe visual loss. Biomicroscopy showed arterialization of the conjunctival vessels and the CAT scan disclosed a dilatation of the superior orbital vein. She was immediately subjected to a neuroradiological fistula closure with recovery of visual acuity to $1.0(20 / 20)$. Our main purpose is to emphasize the importance of the opportune diagnosis and treatment of these fistulas to prevent visual loss and eventual blindness, as occurred in our first patient (Rev Méd Chile 2004; 132: 1221-6).

(Key Words: Carotid-cavernous sinus fistula; Tomography, x-ray computed; Visual acuity)

Recibido el 1 de marzo, 2004. Aceptado el 27 de mayo, 2004.

Servicio de Oftalmología, Hospital del Salvador. Unidad Docente de Oftalmología, Hospital del Salvador, Universidad de Chile.

$\mathrm{S}_{\mathrm{s}}^{\mathrm{e}}$ denomina fístula carótido-cavernosa (FCC) a cualquier comunicación anómala que se establece entre la arteria carótida y el seno cavernoso generando un shunt arteriovenoso patológico, capaz de manifestarse en forma anterógrada a la órbita, produciendo un compromiso ocular severo. Podemos distinguir dentro de este grupo de fístulas dos entidades evolutivas y etiológicas

Correspondencia a: Dr. Raimundo Charlin E, Av. Salvador 300. Providencia, Santiago, Chile.

E-mail: oftalmohsalvador@yahoo.com distintas: 1) Las de tipo directo, que son de alto flujo. En ellas se presenta una comunicación directa entre el tronco principal de la arteria carótida interna y el seno cavernoso. Normalmente están relacionadas a traumatismos severos con fracturas de base de cráneo, pero se han asociado también a traumas quirúrgicos directos, roturas de aneurismas carotídeos intracavernosos, disecciones arteriales o síndromes de deficiencia del colágeno; 2) Existen las fístulas de tipo indirecto, de bajo flujo, que también se conocen como fístulas durales ya que carecen de un factor 
etiológico claro, relacionándose con ciertas malformaciones de la duramadre, donde se establecería una comunicación entre las arterias meníngeas (ramas de la arteria carótida externa e interna) y el seno cavernoso; aquí la porción intracavernosa de la arteria carótida interna permanece intacta. Habitualmente ocurren en forma espontánea, sin embargo, se han asociado a factores predisponentes como hipertensión arterial, trauma, cirugía intracraneal, maniobras de Valsalva, sinusitis y el embarazo $^{1-3}$. Esta clasificación es importante al momento de tomar una conducta terapéutica, debido a que se sabe que más de $50 \%$ de las fístulas de bajo flujo se resuelven espontáneamente ${ }^{4}$, siendo en estos casos la observación la conducta lógica a seguir; en cambio, las de alto flujo requieren de una conducta más agresiva, mediante la embolización vía catéteres intravasculares ${ }^{5}$.

Hemos querido comunicar 2 casos de la experiencia recogida en los últimos dos años en el Servicio de Oftalmología del Hospital del Salvador, donde nos ha tocado diagnosticar y tratar, en conjunto con el Instituto de Neurocirugía, a pacientes con esta patología, poniendo especial énfasis en las diferencias clínicas y de evolución y pronóstico visual de estas fístulas según sea el tipo al que pertenezcan.

\section{PACIENTES y MÉTOdOS}

Caso 1. Paciente de 69 años de sexo masculino que presentó una historia de 2 meses de evolución con aumento de volumen bipalpebral y ojo rojo profundo OD, al cual se agregó, una semana previo a su ingreso, diplopia y limitación de la motilidad ocular. Este paciente había consultado inicialmente en su Servicio de Urgencia, donde se sospechó una celulitis orbitaria, siendo enviado con este diagnóstico y bajo tratamiento antibiótico intravenoso a nuestro Servicio. Al ingreso destacaba en su historia médica una diabetes mellitus de reciente diagnóstico, bien compensada, y el antecedente de un trauma facial con fractura maxilar 20 años antes. Al examen presentaba agudeza visual 0,5 en OD y 1,0 en OI, la tensión ocular era normal, existía una proptosis del ojo derecho con limitación importante de la motilidad ocular y diplopia en todas las direcciones de mirada. Además presentaba una quemosis (edema de conjuntiva) importante del fondo de saco conjuntival inferior, los reflejos fotomotores directo y consensual eran normales y no se observaba defecto pupilar aferente relativo (DPAR). Al examen biomicroscópico destacaba una muy sui generis arterialización de los vasos conjuntivales ( wasos en tirabuzón») (Figura 1). El resto del

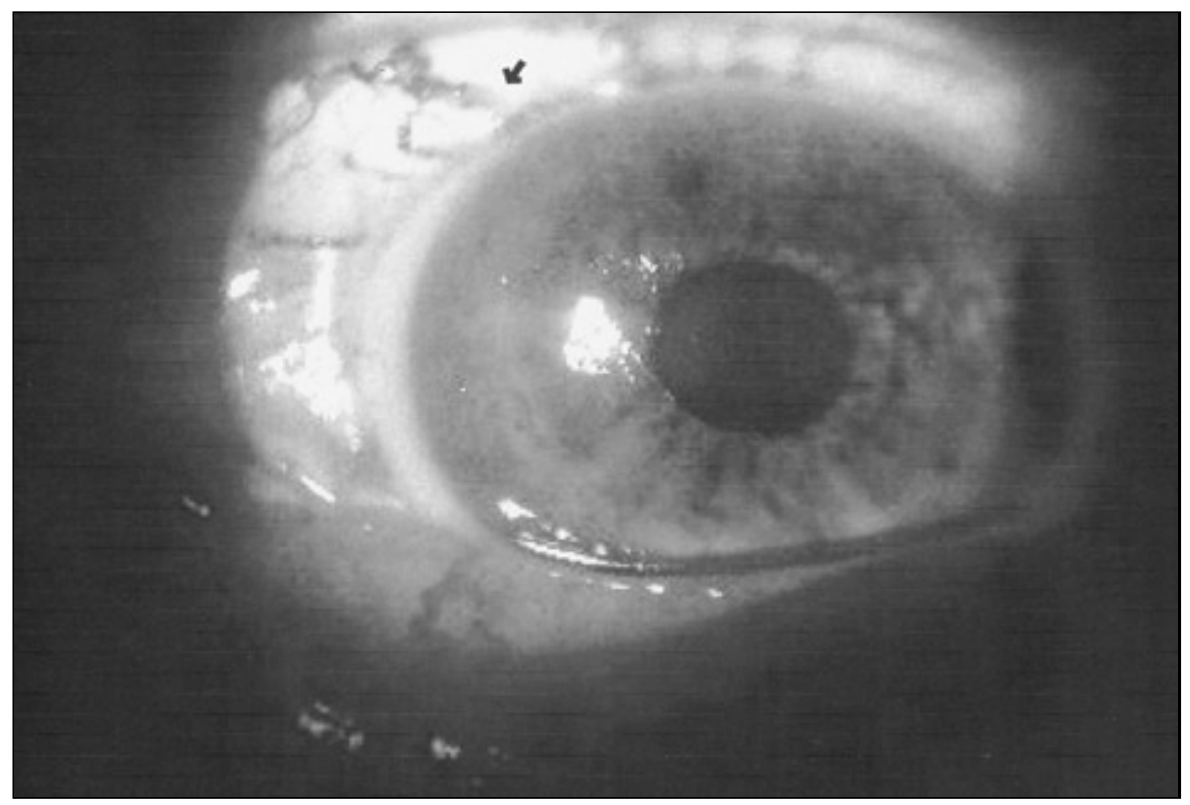

FIguRA 1. Ojo de paciente con fístula carótido cavernosa, se observan quemosis y vasos en tirabuzón (flecha). 
examen ocular y sistémico era normal. Los diagnósticos diferenciales planteados al ingreso fueron: pseudotumor inflamatorio y fístula carótido-cavernosa de bajo flujo, descartándose la celulitis orbitaria por la falta de respuesta a antibióticos y por el tiempo de evolución que el cuadro presentaba. Para diferenciar entre los diagnósticos planteados decidimos iniciar una prueba terapéutica con prednisona en altas dosis durante $48 \mathrm{~h}$, mientras esperábamos la llegada del escáner orbitario. No hubo respuesta a los corticoides en altas dosis y el escáner orbitario mostró una dilatación de la vena orbitaria superior, característica de una fístula carótido cavernosa (Figura 2). De inmediato se solicitó evaluación y tratamiento, vía angiografía, al Instituto de Neurocirugía. Durante las tres semanas de espera del procedimiento neurorradiológico el paciente evolucionó muy mal desde el punto de vista oftalmológico, con disminución progresiva de la agudeza visual, proptosis en aumento, y aparición de DPAR. Al momento en que se realizó el cierre con balón endoluminal de la fístula, el paciente evolucionó con regresión de la proptosis y de la quemosis, sin embargo, la agudeza visual no se recuperó, llegando a visión cero por daño irreversible del nervio óptico por neuropatía óptica compresiva.

Caso 2. Paciente de sexo femenino de 39 años, sana, sin historia de traumatismos encefalocraneanos. Presentaba un cuadro de 1 mes de evolución que se inició con cefalea ocasional leve y acúfenos en el oído derecho. Dos semanas antes de su llegada a nuestro servicio, se agregó proptosis del OD de comienzo súbito por lo cual fue hospitalizada en su ciudad de origen donde se inició tratamiento con prednisona en altas dosis, no respondiendo a esta terapia, razón por la que nos fue derivada. A su ingreso presentaba agudeza visual cuenta dedos a 1 metro en OD y 1,0 en OI, la presión intraocular era de $39 \mathrm{~mm} / \mathrm{Hg}$ en OD y 12 $\mathrm{mm} / \mathrm{Hg}$ en OI. Al examen del OD destacaba una ptosis severa, motilidad ocular restringida, pupila en semimidriasis con reflejo fotomotor disminuido, extensa quemosis inferior, frémito a la palpación de la órbita y un soplo audible al auscultar la órbita derecha. La biomicroscopia evidenciaba los sasos

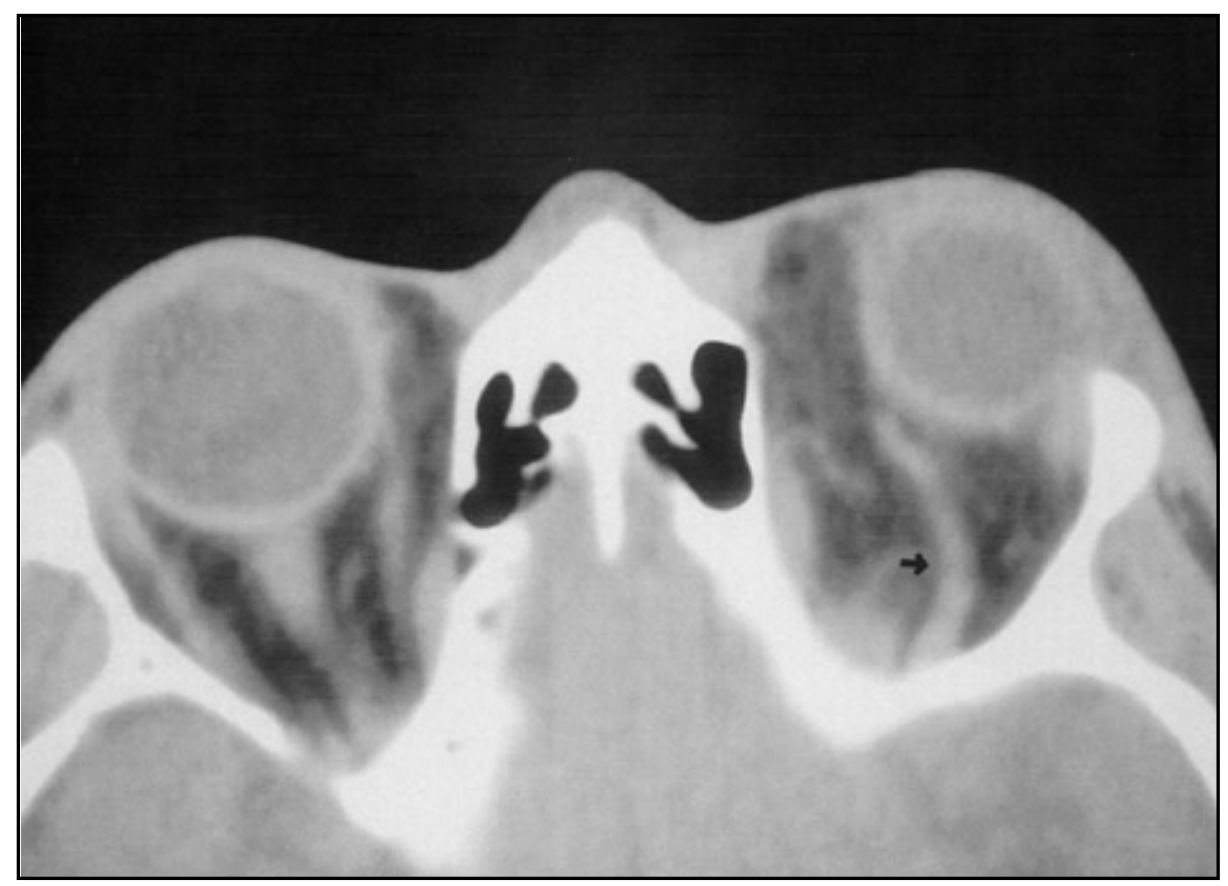

FiguRa 2. Escáner de órbita en paciente con fístula carótido cavernosa, se observa exoftalmo y dilatación de vena orbitaria superior (flecha). 
en tirabuzón»y el fondo de ojo un edema macular discreto del ojo comprometido. Se ingresó con diagnóstico de fístula carótido cavemosa. El escáner evidenció la proptosis con engrosamiento de los músculos extraoculares y la vena oftálmica superior derecha dilatada. En $24 \mathrm{~h}$ fue intervenida neurorradiológicamente, constatándose una fístula de alto flujo en una arteria trigeminal primitiva persistente en el seno cavernoso, realizándose cierre de ésta con mini coils. Evolucionó satisfactoriamente, con mejoría de la agudeza visual a visión $1,0(20 / 20)$, reducción de la presión intraocular a niveles normales y regresión absoluta de su proptosis y quemosis (Figura 3).

\section{Discusión}

Las manifestaciones oculares de las fístulas carótido cavernosas son múltiples y deben alertar a los clínicos sobre la presencia de un shunt patológico. Estas incluyen proptosis, quemosis, congestión epiescleral con vasos en tirabuzón, pulsación del globo ocular, frémito orbitario, soplo orbitario, disminución de la agudeza visual y aumento de la presión intraocular ${ }^{6}$. Se producen debido a la presión venosa elevada que se transmite desde el seno cavernoso hacia la órbita a través de la vena orbitaria superior y sus tributarias.

En el caso de nuestro primer paciente nos enfrentamos a una fístula de bajo flujo, que se fue manifestando solapadamente, lo que produjo mucha dificultad en el diagnóstico desde un primer momento. El hecho de que fuera de bajo flujo no justificaba una larga espera para aplicar un tratamiento efectivo debido a que las manifestaciones oculares eran graves y requerían una resolución oportuna. De lo anterior podemos deducir que una fístula de bajo flujo no es sinónimo de benignidad, al menos para función visual, y la sospecha clínica es muy importante ya que sus manifestaciones pueden ser menos espectaculares que en las de alto flujo. En nuestro caso no había soplo, ni frémito, pero estaba el importante antecedente de un traumatismo craneofacial severo, que siendo más propio de las fístulas de alto flujo, también puede ser causa de las de flujo bajo. $\mathrm{Si}$ bien este primer caso no se manifestó de una manera típica, lo lamentable es que no se logró resolver adecuadamente, en forma oportuna, por la demora en el estudio imagenológico y posterior cirugía, y el ojo terminó ciego. Aunque existen

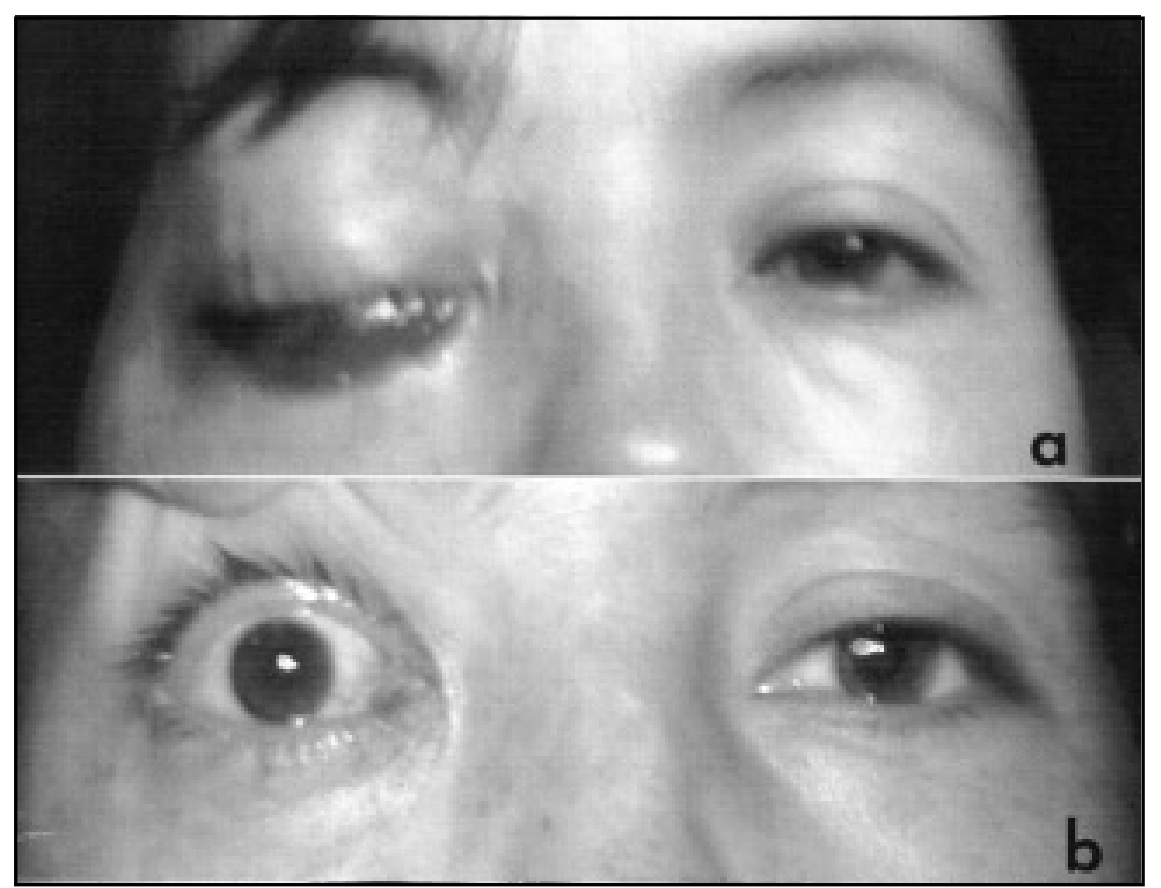

Figura 3. Paciente en pre (a) y post (b) tratamiento endovascular de fístula carótido cavernosa de alto flujo. 
casos descritos de recuperación total de ojos ciegos por esta causa luego del tratamiento endovascular ${ }^{7}$, ello no ocurrió en este paciente. El glaucoma neovascular que se desarrolló posteriormente, es una complicación rara y se debió probablemente a la congestión venosa y a la isquemia retinal secundaria, ya que no existían elementos clínicos de trombosis venosa retinal ni de oclusión arterial ${ }^{8}$.

Por otra parte, en nuestro segundo caso, se observó un cuadro típico de fístula carótidocavernosa de alto flujo que permitió un diagnóstico y tratamiento oportunos, lográndose una recuperación satisfactoria tanto anatómica como funcional.

La arteria trigeminal primitiva está normalmente presente durante 7 días en el desarrollo fetal y actúa como anastomosis entre los sistemas carotídeo y vertebral. Su persistencia constituye el más frecuente remanente embriológico del sistema vascular cerebral, y puede predisponer al desarrollo de FCC frente a traumas craneanos insignificantes ${ }^{9}$.

En relación al diagnóstico diferencial, es importante destacar la utilidad de la prueba con corticoides en altas dosis puesto que es muy efectiva en revertir los signos y síntomas en casos de orbitopatía distiroidea y pseudotumor inflamatorio y no así en las celulitis orbitarias o en las FCC. Su uso se encuentra ampliamente avalado ${ }^{10}$. En el correcto diagnóstico diferencial debemos considerar también todas las manifestaciones sistémicas que acompañan a la orbitopatía distiroidea (temblor, diarrea, sudoración, astenia) y a la celulitis orbitaria (compromiso del estado general, fiebre, dolor), apoyados por un estudio de laboratorio completo (hemograma, hemocultivos, pruebas tiroideas), lo que nos permitirá iniciar el tratamiento adecuado.

Descartadas las causas más frecuentes de exoftalmos que ya hemos señalado, y si estamos considerando el diagnóstico de FCC al momento de decidir las opciones terapéuticas, es importante

\section{REFERENCIAS}

1. Barrow D, Spector R, Braun I. Classification and treatment of spontaneous carotid cavernous fistula. J Neurosurg 1985; 62: 248-56.

2. Keltner J, Satterfield D, Dublin A, Lee B. Dural and considerar si la fístula es de alto o bajo flujo a la arteriografía. Respecto de las de alto flujo no existe duda en que el tratamiento endovascular con microbalones, mini coils o agentes embolígenos debe ser de elección ${ }^{1-3,11}$. En las de bajo flujo, clásicamente se ha considerado la observación con un seguimiento a largo plazo como una medida lógica a seguir. Sin embargo, últimamente se ha descrito la existencia de un drenaje venoso cortical retrógrado anómalo ${ }^{11}$ asociado a este tipo de fístulas que se manifiesta con déficit neurológico, severas cefaleas, hemorragias intradurales, trombosis venosas, estados mentales alterados y amenaza de la función visual, que pueden estar presentes hasta en el $55 \%$ de los casos y que justificaría el riesgo de tratar las fístulas de bajo flujo con métodos endovasculares con una tasa de éxito de $90 \%$ y con complicaciones serias relativamente bajas (esta decisión debe ser tomada en conjunto con el paciente). Por otro lado, si la fístula de bajo flujo es de escaso riesgo, con sintomatología leve, y sin amenaza de la función visual, puede no requerir intervención activa y puede ser manejada en forma expectante en espera de un posible cierre espontáneo ${ }^{11}$. Sin embargo, en casos en que la visión esté amenazada, como en nuestro primer paciente, es imperativo acelerar el tratamiento para no terminar con un desenlace tan negativo como es la ceguera del ojo afectado ${ }^{5}$.

Si bien la clasificación en alto y bajo flujo muestra fenómenos hemodinámicos y anatómicos disímiles para ambos tipos de fístulas, la expresión clínica de ellas carece de elementos claramente objetivos que permitan una diferenciación definitiva entre éstas, siendo el estudio angiográfico fundamental para su correcta clasificación y para un adecuado y oportuno tratamiento. Queremos, por lo tanto, enfatizar la importancia del correcto diagnóstico diferencial que esta patología merece, debido a las devastadoras consecuencias oculares que un tratamiento tardío conlleva.

carotid cavernous fistulas. Diagnosis, management and complications. Ophthalmology 1987; 94: 1585-99.

3. Macho J, Guelbenzu S, Barrena R, Valies V, Ibarra B, VALERo P. Carotid cavernous fistula: endovascular therapy. Rev Neurol 1996; 24: 59-64. 
4. Chavis R, Krohel G, Perman K. Acute proptosis in adults. En: Tasman W, ed. Duane's clinical ophthalmology, vol 2, chap 28, Philadelphia, Lippincott-Raven publishers, 1997; pp 14.

5. Halbach V, Hieshima G, Higashida R, Reicher M. Carotid cavernous fistula: indications for urgent treatment. Am J Roentgenol 1987; 149: 587-93.

6. DurCan F. Carotid cavernous fistula. En: Gold D, Weingest $\mathrm{T}$, eds. The eye in systemic disease. Philadelphia, Lippincott, 1990; pp 658-60.

7. Brodsky M, Hoyt W, Halbach V, Hieshima G, Higashida R, Barbaro N. Recovery from total monocular blindness after ballon embolization of carotid cavernous fistula. Am J Ophthalmol 1987; 104: 86-7.
8. Gupta N, KiKKAWA D, Levi L, WeinReB R. Severe vision loss and neovascular glaucoma complicating superior ophthalmic vein approach to carotid cavemous fistula. Am J Ophthalmol 1997; 124: 853-5.

9. Cook B, Leavitt J, Dolan J, Nichols D. Carotid cavernous fistula associated with persistent primitive trigeminal artery. J Neurophthalmol 2000; 20: 264-5.

10. Krohel G, Stewart W, Chavis RM. Orbital disease: a practical approach. New York, Grune \& Stratton, 1981; pp 27-9.

11. Meyers P, Halbach V, Dowd C, Lempert T, Maiek A, Phatouros C et al. Dural Carotid cavernous fistula: definitive endovascular management and long term follow up. Am J Ophthalmol 2002; 134: 85-92. 